\title{
CATÁLISIS ASIMÉTRICA, UNA NUEVA ERA EN LA SÍNTESIS DE FÁRMACOS: HISTORIA Y EVOLUCIÓN
}

\author{
ASYMMETRIC CATALYSIS, A NEW ERA IN THE SYNTHESIS OF DRUGS: HISTORY AND EVOLUTION \\ James O GUEVARA-PULIDO²*, Jennifer CAICEDO', Fredy DAVID', Miguel \\ VELA', Jhon GONZÁLEZ ${ }^{2}$ \\ ${ }^{1}$ PCLQ, Universidad Distrital Francisco José de Caldas, Bogotá-Colombia \\ 2 Universidad El Bosque, Bogotá-Colombia. \\ *Autor Corresponsal. E-mail: joguevara@unbosque.edu.co \\ Historia del Artículo \\ Recibido: Julio 27, 2016 \\ Evaluado: Noviembre 15, 2016 \\ Aceptado: Enero 16, 2017 \\ Disponible: Febrero 09, 2017
}

Resumen |

El entendimiento de la Química Orgánica a lo largo del tiempo, lleva a los científicos a proponer estrategias sintéticas que permiten la obtención de compuestos de interés biológico de manera enantio y diastereoselectiva. La preparación de estructuras quirales de forma enantiopura ha sido, desde la antigüedad, un logro que han pretendido alcanzar gran cantidad de químicos sintéticos. Inicialmente, se desarrollaron gran cantidad de transformaciones diastereoselectivas, las cuales implican que a partir de reactivos enantioméricamente puros (o creados de forma temporal durante la reacción, empleando sustancias auxiliares o plantillas quirales), es posible inducir la reacción en el sentido estéreo deseado teniendo como base los estereocentros existentes en estos compuestos de partida. También se conocen reacciones en donde se pueden obtener enantiómeros puros a partir de moléculas de origen natural, que poseen estereoquímica próxima a la deseada (chiral pool). Estos métodos aunque ofrecen algunas ventajas de síntesis presentan varios inconvenientes. En las últimas décadas, han ganado relevancia los métodos catalíticos enantioselectivos, que solucionan en gran parte los inconvenientes presentados en las otras metodologías. Se pueden clasificar en tres grandes grupos, totalmente complementarios: catálisis por metales, catálisis enzimática y organocatálisis. Está revisión pretende dar una visión general de los aportes de cada uno de los métodos de síntesis, profundizando en la organocatálisis, la estrategia más eficiente en la actualidad.

Palabras Clave: Enzimas, Organometálico, Organocatálisis, Enantioselectividad, Diastereoselectividad.

\section{Abstract |}

The organic chemistry knowledge over time has prompted scientists to propose new Enantio- and Diastereoselective synthesis in order to obtain compounds of biological interest. The preparation of enantipure chiral structures has been, since ancient times, an achievement that a large number of synthetic chemists have attempted to reach. Initially, a large number of diastereoselective transformations were develop, these methods imply that from enantiomerically pure reagents (or temporarily created during the reaction, using auxiliary chiral substances or templates), were possible to induce the desired stereo reaction based on the existing stereocenters in these starting compounds. In addition, reactions where was probable obtain pure enantiomers from compounds of natural origin, which have stereochemistry too close to the desired (chiral pool), were use. These methods offer some advantages of synthesis, but presents several drawbacks. The enantioselective catalytic methods became particularly important during the last decades because solve in great measure the problems presented in the other methodologies. They classified in three big groups, fully complementary: catalysis by metals, enzyme catalysis and organocatalysis. This review aims to give an overview of the contributions from each method of synthesis, especially in the organocatalysis, the most efficient strategy in the present.

Keywords: Enzymes, Organometallic, Organocatalysis, Enantioselectivity, Diastereoselectivity.

\section{INTRODUCCIÓN |}

En la actualidad aproximadamente el $56 \%$ de los medicamentos disponibles en el mercado cuenta con al menos un centro quiral; de estos, el $86 \%$ se comercializan como mezclas racémicas. Por esta razón, la síntesis orgánica moderna se ha enfocado en la síntesis asimétrica o síntesis enantioselectiva; esta se ha desarrollado en tres pilares catalíticos: la biocatalisis, la catálisis organometálica y la organocatálisis. Estos pilares difieren entre sí según el tipo de catalizador utilizado para inducir la enantioselectividad de la reacción (enzimas, compuestos órganometalicos y 
compuestos orgánicos respectivamente). Cada una de estas categorías se ha desarrollado con distinta intensidad a través de los años a partir del reconocimiento de la importancia de la enantio y estereoselectividad en la obtención de productos orgánicos con propósitos industriales y farmacéuticos. De acuerdo con lo anterior, este artículo tiene como propósito dar una visión histórica y moderna de las contribuciones más relevantes que se han generado en pro del fortalecimiento de la síntesis asimétrica, tratando de establecer la actualidad de esta metodología sintética en Latinoamérica.

\section{BIOCATÁLISIS O CATÁLISIS ENZIMÁTICA}

En la naturaleza los productos de síntesis como carbohidratos, lípidos, aminoácidos y los compuestos biológicamente activos (como los neurotransmisores y hormonas) se obtienen y actúan de forma enantiomérica. Estos deben ser altamente específicos debido a que intervienen en los procesos biológicos y en la información de los mismos, que es controlada por mensajeros que interactúan selectivamente en sitios particulares de enzimas, receptores, moléculas transportadoras, etc., y cuya interacción depende de la complementariedad química entre estos agentes quirales. Esta perfección sintética es deseada por los procesos sintéticos industriales y farmacológicos, ya que permite reducir costos de separación de enantiómeros y evita los efectos secundarios producidos por el enantiómero homólogo al activo; de acuerdo con esto, desde los inicios las enzimas representaron una alternativa lógica para desarrollar procesos de síntesis mucho más rentables y específicos. (Perotti, 2012)

Los catalizadores de naturaleza enzimática han sido utilizados por los seres humanos durante miles de años, por ejemplo, en la fermentación como un medio para producir y conservar los productos alimenticios como el queso, la cerveza, el vinagre y el vino (Reetz, 2013). No fue sino hasta hace un poco más de 100 años que estos catalizadores empezaron a utilizarse con propósitos académicos específicos, el ejemplo más icónico para la época fue el tratamiento que Pasteur, en 1858 , le dio a una solución acuosa de sal de amonio del ácido tartárico racémico mediante una acción realizada por el hongo Penicillium glaucum. Esto conllevó a una resolución de la mezcla racémica obteniendo el ácido (-) tartárico mientras que el ácido (+) tartárico se descomponía. Pasteur ya reconocía la existencia de moléculas asimétricas, dado que en sus memorias escribió: "La mayoría de los productos naturales, los productos esenciales para la vida son asimétricos y poseen tal asimetría que sus imágenes no son superponibles. Esto establece tal vez la única línea de demarcación que al presente puede trazarse entre la química de la materia sin vida y la química de la materia viviente" (Perotti, 2012). La resolución bioquímica (como también se le conoce) que Pasteur realizó, contribuyó de forma tan significativa que hoy en día se considera un precursor de la resolución cinética catalizada por enzimas (Reetz, 2013; Beyer y Walter, 1987). Más adelante, científicos como Emil Fischer, Albert Eschenmosser, Friedrich Lichtenthaler y Linus Paulling realizaron investigaciones que contribuyeron al origen de la biocatálisis; pero fue Eduard Buchner en 1897 quien derrumbó el paradigma asociado a la necesidad de usar células vivas para poder llevar a cabo procesos catalizados, dando paso a la biocatálisis como la conocemos hoy en día y abriendo las posibilidades a innumerables procesos académicos e industriales donde se utilizaban extractos celulares o enzimas parcialmente purificadas con propósitos biocatáliticos. (Reetz, 2013)

Luego de 16 años, Ludwig Rosenthaler describe en 1913 la síntesis del ( $R$ )-mandelonitrilo mediante la reacción del benzaldehído con $\mathrm{HCN}$ en presencia de un extracto de almendras, la cual contenía una mezcla de enzimas oxinitrilasas las cuales en la actualidad se comercializan y permiten un exceso enantiomérico (ee) $>95 \%$. (Figura 1).

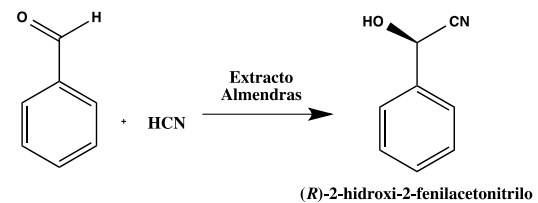

Figura 1. Síntesis del (R)-mandelonitrilo a partir del benzaldehído y HCN (Reetz, 2013).

A mediados de 1950, compañías farmacéuticas como Upjohn, Schering, Pfizer, y Merck, empezaron a desarrollar procesos de hidroxilación oxidativa regio- y estereoselectiva de esteroides mediante el uso de cepas microbianas, comercializando entonces medicamentos como la cortisona y la hidrocortisona; a pesar de esto, la creación de procesos para el aislamiento, purificación y optimización de enzimas fue desarrollada hasta diez años después, lo que coincidió con la investigación en las vías metabólicas; un ejemplo de esta optimización fue la desarrollada por Alexander Klibanov, quien demostró que algunas lipasas conservan su actividad en solventes orgánicos, ofreciendo una alternativa a las reacciones que presentaban inconvenientes para realizarse en medios acuosos (Reetz, 2013).

Pese al creciente avance científico, la biocatálisis no lograba superar su mayor barrera, para 1970 la producción en masa de proteínas no era posible dificultando así un extendido uso industrial; por otro lado, la extracción de enzimas de hongos, animales, plantas e inclusos insectos, eran procesos costosos y a menudo complicados lo que impedía el reconocimiento de estos procesos en la Química Orgánica. Esta desventaja de la biocatálisis fue superada gracias a los estudios de Paul Berg, Herbert Boyer y Stanley Cohen, quienes desarrollaron una metodología de ADN recombinante (Beyer y Walter, 1987) en la cual una determinada enzima que se produce en un organismo puede ser sobreexpresada en otro organismo huésped, lo que permitió obtener grandes cantidades de enzimas, útil para su extrapolación a uso industrial como en la de la fabricación de 
detergentes producidos por empresas como Henkel (Alemania) o Novozyme (Dinamarca).

Otras técnicas surgieron como sistemas de expresión alternativos: ingeniería biotecnológica de reactores e inmovilización de enzimas, para aquellos en los que el ADN recombinante no comprendía una opción viable. Sin embargo, la técnica que representó mayor importancia fue la propuesta por Kary Mullis en 1983, la cual consistió en la reacción en cadena de la polimerasa (PCR), técnica que en la actualidad es de gran importancia en la biología molecular. Para el año 1990, la síntesis de productos enantioméricamente puros se realizaba en mayor proporción, además mediante procesos bio-catalíticos se empezaban a desarrollar medicamentos como antibióticos, sobre todo derivados 8 -lactámicos como la cefalexina. Otro ejemplo de la producción industrial consiste en la hidrólisis parcial del acrilonitrilo para la formación de acrilamida ultrapura que es catalizada por una nitrilo hidratasa, esta reacción es desarrollada por una empresa Japonesa llamada Nitto Chemical Industry Company, la cual presenta una capacidad de $4 \times 10^{5}$ toneladas por año (Figura 2). (Reetz, 2013)

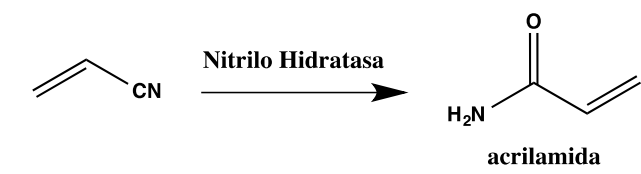

Figura 2. Síntesis de acrilamida (Reetz, 2013).

A pesar de la evidente importancia que tuvieron las enzimas para el desarrollo de procesos industriales, estás todavía presentaban ciertas desventajas tales como la estereoselectividad insuficiente, pobre regioselectividad, la falta de estabilidad en algunas condiciones de funcionamiento (altas o bajas temperaturas) y en ocasiones hasta la inhibición del producto. Científicos como Michael Smith, intentaron mejorar algunas de estas complicaciones con la sustitución de algunos aminoácidos presentes en estas proteínas, abriendo así el camino para la futura manipulación de la estructura y propiedades catalíticas de las enzimas. Es aquí donde surge en Alemania, desde el Laboratorio de Mülheim a cargo del Profesor Manfred T. Reetz, la evolución dirigida o también llamada evolución de laboratorio, la cual ha permitido modificar a conveniencia las enzimas mediante ciclos repetidos de mutagénesis de genes por ejemplo reacción en cadena de la polimerasa propensa a errores (epPCR), mutagénesis de saturación y además con técnicas de expresión y selección imitando así la evolución natural (Reetz, 2010). De esta forma se hacen más termoestables, en principio, y luego mejoran su enantioselectividad tras la sustitución y recombinación de aminoácidos de la proteína mediante métodos de PCR. Entre los estudios iniciales de este grupo de investigación, se encuentra la mejora de la enantioselectividad de la enzima lipasa de Pseudomonas aeruginosa (bacterias Gram-negativas) denominada PAL, para utilizarse como un catalizador para la resolución cinética hidrolítica del $p$-nitrofenilester (Figura 3). El factor de selectividad (E) para el enantiómero $\boldsymbol{R}$ fue aumentando acorde con el número de PCR que se le realizaron a la enzima, ya que de esta manera fue posible encontrar la cadena de aminoácidos que comprendían el sitio activo de la enzima, lo que mejoró considerablemente la estereoselectividad de la reacción. (Reetz et al, 2010).

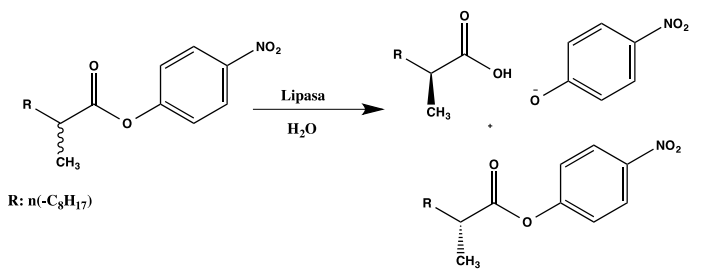

Figura 3. Resolución cinética hidrolítica de esteres catalizada por PAL mutantes. (Reetz, 2010).

Uno de los ejemplos más característicos de la eficacia de la evolución dirigida, consiste en la modificación mediante epPCR de la ciclohexanona monooxigenasa (CHMO), la cual es capaz de catalizar reacciones de sulfoxidación asimétrica de tioéteres proquirales para la formación de sulfóxidos quirales (Figura 4).

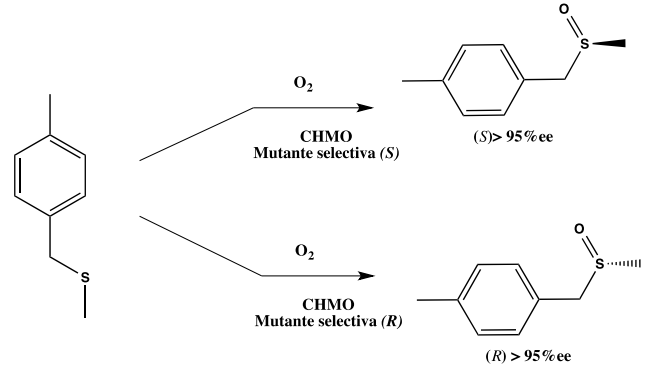

Figura 4. Sulfoxidación enantioselectiva catalizada por CHMO mutante. (Reetz, 2010).

En la actualidad, laboratorios como los del profesor Reetz (Alemania) continúan trabajando en el mejoramiento de la evolución dirigida, ya que hoy en día se considera que aproximadamente el $10 \%$ de las síntesis de fármacos que se comercializan corresponde a procesos de síntesis biocatalizados (Patel, 2008), siendo este un bajo porcentaje en comparación con catalizadores organometálicos. A pesar de la importancia y necesidad que representa el mejoramiento de catalizadores, en este caso enzimáticos, son pocos los países que trabajan en este tipo de modificaciones enzimáticas, entre los cuales se destacan Alemania y España y desde hace un poco más de 5 años México, con estudios de la Universidad Autónoma de México (UNAM), quienes han desarrollado algunos proyectos relacionados con la generación de biocatalizadores.

\section{CATÁLISIS ORGANOMETÁLICA}

La catálisis por compuestos organometálicos ha recibido una creciente atención desde la década de los $70^{\prime}$. Wikilson y sus colaboradores se aproximaron al entendimiento de las reducciones asimétricas a partir de sus estudios de 
hidrogenación catalítica por complejos de $\operatorname{RhCl}\left(\mathrm{PPh}_{3}\right)_{3}$. (Knowles y Sabacky, 1968) usaron fosfinas quirales monodentadas para este proceso, donde obtuvieron resultados decepcionantes, pero que mejoraron tiempo después (Knowles et al, 1972). En 1971, (Dang y Kagan, 1971) prepararon difosfina 2,3-O-isopropiliden-2,3-dihidroxi-1,4bis(difenilfosfino)butano (DIOP) y mediante algunos complejos de Rodio (Rh) resultado de sus trabajos, lograron obtener un exceso enantiomérico (ee) > $70 \%$ en la hidrogenación de olefinas con diferentes grupos funcionales como sustituyentes.

Knowles y colaboradores aportaron al desarrollo a nivel industrial de la obtención de la 3,4-dihidroxifenilalanina (LDOPA), a partir de la etano-1,2-diil-bis[(2-metoxifenil) fenilfosfano] (DiPAMP)(Vineyard et al, 1977) en donde incorporaron algunos complejos catiónicos de Rh obtenidos por Schrock y Osborn. Este proceso comenzó a operar desde 1974 (Figura 5) (Pizzano y Carmona, 2001).

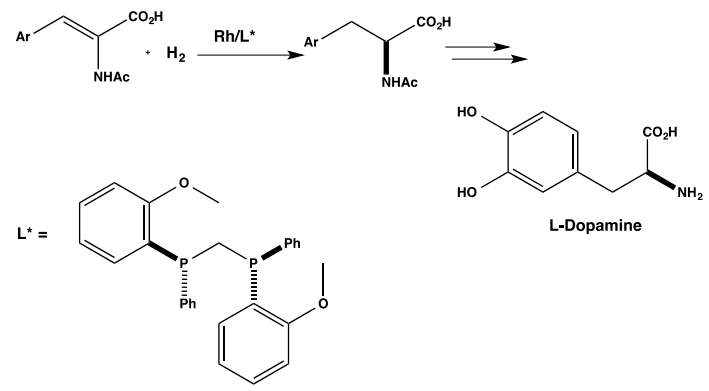

Figura 5. Síntesis de L-DOPA (Pizzano y Carmona, 2001).

Noyori se destaca por su contribución a las reacciones de hidrogenación asimétrica, la primera de ellas consistía en la síntesis de 2,2'-bis(difenilfosfino)-1,1'-binaftilo (BINAP), la aplicación de este ligando fue exitoso dada su versatilidad en distintas reacciones catalíticas, cuya variedad y complejidad aún en día crece (Miyashita, 1980).

La incorporación de Rodio al BINAP ayudó en el desarrollo de diversas reacciones de hidrogenación de olefinas con otros grupos funcionales; pero el mayor éxito se encontró con la incorporación de Rutenio ( $R u$ II), con el que se llegó a hidrogenar diversos compuestos $\alpha, \beta$-insaturados con excesos enantioméricos (ee) muy altos (Ohta, 1987), una de las reacciones a destacar es la síntesis de ácidos $\beta$-aril propiónicos quirales que se usan como anti-inflamatorios (como el Naproxeno). De igual manera, su aplicabilidad se extiende a la reducción de diversos alcoholes alílicos o quinolínicos, reacciones que transcurren con buenos resultados (Takaya et al, 1987). Los compuestos de rutenio también se han utilizado en la hidrogenación de diversas cetonas a los correspondientes alcoholes quirales, como es el caso de las $\beta$-lactamas (Kitamura et al, 1988).

Otro gran aporte de Noyori se encontró en la incorporación de diaminas a los compuestos con Rutenio, con lo cual la quimio-selectividad a la formación de alcoholes insaturados aumentó (Ohkuma, 1995). Por sus estudios en transferencia de hidrógeno (Noyori, 1997) y sus contribuciones a las adiciones asimétricas, Noyori recibió gran reconocimiento científico a nivel internacional (Noyori y Kitamura, 1991).

Barry Sharpless es otro investigador que contribuyó a la catálisis organometálica, profundizando esencialmente en las reacciones de oxidación; su esfuerzo se basó en controlar la enantioselectividad en la epoxidación de olefinas, estudios que iniciaron en 1965, y que ya por 1980 , con ayuda de Katsuki, los resultados dieron frutos, al obtenerse alcoholes alílicos con excelentes enantioselectividades al utilizar como catalizador isopropóxido de titanio $\left(\mathrm{Ti}(\mathrm{OPri})_{4}\right)$ con tartrato de dietilo (Katsuki y Sharpless, 1980), (Janice et al, 1987). Este avance permitió que se pueda sintetizar glicidol a escala industrial, compuesto que se utiliza como intermediario en la fabricación de $\beta$-bloqueantes. (Pizzano y Carmona, 2001).

Sharpless también trabajo y realizó aportes en la dihidroxilación de olefinas catalizadas por compuestos de Osmio. Logro obtener una amplia gama de dioles quirales a través de un sistema quiral con la Dihidridoquinidina (DHQD o DHD), y también preparó diferentes ligandos quirales a partir de aminas, consiguiendo optimizar el proceso de dihidroxilacion, en donde reemplazó el Tetróxido de Osmio $\left(\mathrm{OsO}_{4}\right)$ con Osmato de Potasio y haciendo uso de sistemas bifásicos en su reacción, convirtió a esta en uno de los procesos enantioselectivos de mayor uso actualmente (Kolb et al, 1994).

Las labores, trabajos y logros alcanzados en el campo de la catálisis organometálica, se vieron recompensados con el premio de Nobel de Química del año 2001, otorgado a los científicos Karl Barry Sharpless, William S. Knowles y Ryōji Noyori.

Entre otros avances relacionados con la catálisis mediada por compuestos organometálicos, se mencionan los trabajos de Shibashaki y colaboradores, cuando en 1997, describieron una reacción aldólica promovida por catalizadores metálicos bifuncionales, imitando a las aldolasas de tipo II. (Yamada y Shibasaki, 1997). Desde entonces, desde el grupo de Shibashaki, han desarrollado diferentes sistemas catalíticos, caracterizados principalmente por tener dos o más centros de coordinación ácido y básico, lo que hoy en día se conoce como catalizadores multifuncionales (Figura 6) (Shibasaki et al, 2009; Matsunaga y Shibasa, 2008).

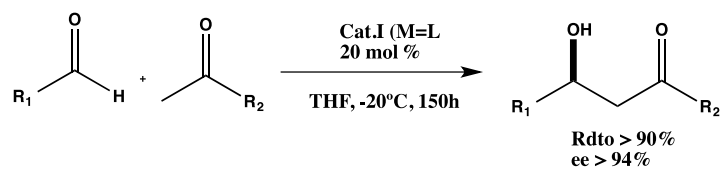

Ligando LLB

Figura 6. Reacción aldólica de Shibashaki, catalizada por $\mathrm{LaLi}_{3}$ tris (binaftóxido) (LLB), alcanzando 94\% ee, que antes solo era logrado por las enzimas. El catalizador imita la función de la enzima mostrando tanto la acidez de Lewis y la basicidad Brønsted. M: Li, $\mathrm{Na}, \mathrm{K}$; Ln: lantánido. (Shibasaki et al, 2009). 


\section{ORGANOCATÁLISIS}

La organocatálisis es una estrategia reciente en la síntesis asimétrica, con respecto a otros campos como los son la catálisis enzimática y organometálica. En esta, se hace uso de moléculas orgánicas de bajo peso molecular que actúan como catalizadores, todo con el fin de conseguir transformaciones químicas altamente enantioselectivas. Aunque ya se habían presentado reacciones catalizadas por moléculas orgánicas, no fue sino hasta la década de los 90 donde se empezaron a documentar nuevos hallazgos y exploraciones en dicho campo. Esto se ve reflejado en al menos 1500 artículos, que describen el uso de organocatalizadores en más de 130 tipos de reacciones, publicadas entre 1998 y 2008 (Macmilan, 2008). Cabe aclarar que entre 1968 y 1997, se publicaron reacciones catalizadas con este tipo de compuestos, pero no se les daba el enfoque asimétrico ni su posible aplicación potencial en otras reacciones, solo se resaltaban los resultados obtenidos en pocos procesos. Esto llevo a que la catálisis por moléculas orgánicas pasara desapercibida durante un periodo de tiempo prolongado $y$, por consiguiente, que no fuese considerada un campo de estudio sobresaliente.

Hasta finales de la década de los noventa, surgieron algunos autores y grupos de investigación que lograron generar síntesis enantioselectivas usando como catalizadores compuestos orgánicos, es el caso de (Tu et al, 1996; Denmark et al, 1997; Yang, 1996) quienes demostraron que las cetonas enantioméricamente puras podían utilizarse para catalizar la epoxidación enantioselectiva de alquenos simples. También en este tiempo, llegaron los primeros ejemplos de catálisis por puentes de hidrógeno, en una reacción conocida como Strecker asimétrica (Sigman y Jacobsen, 2000; Corey y Grogan, 1999) y junto a esto, la introducción del concepto de péptidos mímicos para la resolución cinética enantioselectiva de alcoholes, por (Miller, 1998). Aunque estos trabajos no fueron el inicio de la organocatálisis como un campo de investigación, si permitieron resolver problemas de la síntesis química de aquella época. Sin embargo, solo hasta el año 2000 se publicaron dos investigaciones basadas en las enaminas (List et al, 2000) y los iones iminio (Macmilan, 2008), tomados como punto de partida para el uso de la organocatálisis como estrategia sintética. Con los trabajos de Barbas y sus colaboradores, se logró demostrar una ruta sintética alternativa a la reacción de Hajos-Parrish, esto conllevo a que se entendiera y aplicaran dichos avances en reacciones aldólicas intermoleculares. Por otra parte este trabajo también demostró que a partir de moléculas orgánicas pequeñas, como la prolina, se pueden realizar síntesis de carácter enzimático, en donde se reemplazan moléculas grandes y complejas por catalizadores más sencillos, obteniendo mecanismos de reacción similares.

Una vez que la organocatálisis toma fuerza como objeto de estudio, se empieza a evidenciar algunas ventajas con respecto a los métodos que antes se habían tratado en la síntesis asimétrica, de las cuales sobresalen la facilidad y el bajo costo de llevar a cabo este tipo de reacciones en el laboratorio y el potencial de las nuevas líneas de pensamiento académico y de investigación (Macmilan, 2008).

Las reacciones con organocatalizadores aportaron una serie de ventajas en este campo, que van desde el ahorro de costos, tiempo y energía, pasando por la simplicidad experimental y por último la reducción de residuos. Estas ventajas se deben a tres factores principalmente: el primero, los catalizadores orgánicos, por lo general, son resistentes al oxígeno y la humedad atmosférica, por lo que no es necesario contenedores herméticos o especiales para su almacenamiento. El segundo, en la naturaleza están presentes una gran variedad de compuestos orgánicos (aminoácidos, carbohidratos, hidroxiácidos, etc.) en forma de enantiómeros individuales, lo que hace que la preparación de los catalizadores orgánicos sea económica, y permite también obtener gran cantidad de estos. Por último, normalmente tiene baja toxicidad $y$ son amigables ambientalmente, lo que aporta una mayor seguridad en el proceso de catálisis, muy útil en cualquier tipo de investigación.

El éxito de la organocatálisis (además de sus múltiples ventajas) radica principalmente en la identificación de los modos genéricos de activación del catalizador, inducción y reactividad. Se entiende como modo genérico de activación a la especie química que tiene la capacidad de participar en muchos tipos de reacción con alta enantioselectividad, en otras palabras la interacción de un solo catalizador quiral con un grupo funcional (cetona, aldehído, alqueno o imina) de manera organizada y predecible (Macmilan, 2008).

Con la aparición de dichos modos de activación, se hace relativamente sencillo el uso de estos como plantillas para el planteamiento de nuevas reacciones enantioselectivas. De hecho, en 1998 se reportaron alrededor de 130 reacciones con solo 5 o 6 modos de activación, lo que es de gran utilidad para los químicos, ya que facilita el desarrollo de catalizadores o familias de catalizadores que son útiles para una amplia gama de reacciones asimétricas. Desde la década de los 70 , se han identificado modos de reacción que catalizan varios procesos de gran interés en el campo de la química que a continuación se resaltarán:

\section{Catálisis por enaminas}

En 1971, se publicaron dos documentos independientes (Hajos y Parrish, 1971; Eder et al, 1971) de una reacción aldólica enantioselectiva intramolecular, catalizada con prolina en la reacción de Wieland-Miescher, que aunque tuvo resultados extraordinarios, no fue explotada sino hasta el año 2000 por (List et al, 2000), desde entonces han surgido una gran cantidad de investigaciones dirigidas a la identificación de nuevos catalizadores que formen enaminas 
quirales. La amina que contiene el catalizador, típicamente interactúa con un sustrato cetona para formar una enamina intermedia, pero a la vez se acopla con una pareja de reacción electrofilica ya sea a través de enlaces de hidrógeno o atracción electrostática (Figura 7).

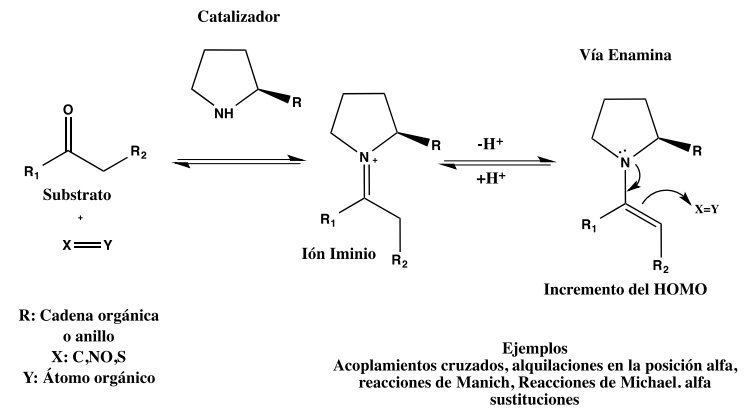

Figura 7. Modo de reacción por vía Enamina (Macmilan, 2008).

\section{Catálisis por puentes de hidrógeno}

A principios de la década de 1980, los investigadores descubrieron varios procesos catalíticos asimétricos, que sugieren que la activación de un sustrato y la organización del estado de transición podrían producirse a través de interacciones de enlace de hidrógeno bien definidos (Macmilan, 2008). Sin embargo, dichos estudios fueron considerados excepciones a la idea que el enlace de hidrógeno era insuficiente para la activación en una catálisis asimétrica. Esto fue refutado en los años 1998 y 1999 por (Sigman y Jacobsen, 2000; Corey y Grogan, 1999), que realizaron por aparte una variante sintética de la reacción de Strecker utilizando catalizadores de enlaces de hidrógeno bien definidos. Cuatro años más tarde, Jacobsen mostró que estos catalizadores (e.g., tiourea) se podrían usar para otras reacciones, proyectando el uso genérico de catálisis enantioselectiva por enlaces de hidrógeno (Figura 8).

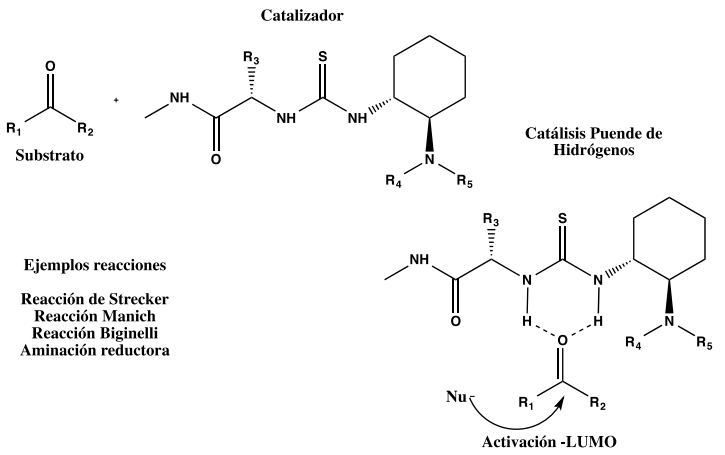

Figura 8. Modo de reacción por vía de puentes de hidrógeno (Macmilan, 2008).

\section{Catálisis vía ión iminio}

Fue el primer modo de activación organocatalítica en ser diseñada y es usada como una estrategia general para la síntesis asimétrica. Se basa en la capacidad de las aminas quirales para funcionar como catalizadores enantioselectivos en varias transformaciones que usan tradicionalmente ácidos de Lewis como catalizadores. El concepto se basa en la hipótesis mecanicista que la formación reversible de iones iminio en aldehídos $\alpha$,ß-insaturados y aminas quirales, podría emular la dinámica del equilibrio y los orbitales $\pi$ electrónicos que son inherentes a la catálisis ácido de Lewis (Figura 9). (Macmilan, 2008).
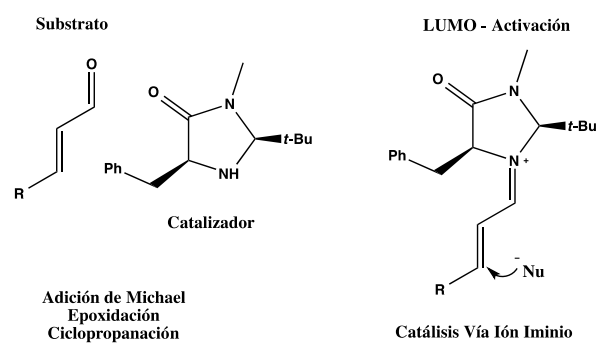

Catálisis Vía Ión Iminio

Figura 9. Modo de reacción vía iminio (Macmilan, 2008).

Algunos ejemplos recientes de este tipo de catálisis utilizando como catalizador un derivado del aminoácido prolina se describen a continuación:

En 2006 Jørgensen y colaboradores publicaron el primer ejemplo de adición enantioselectiva organocatalizada de malonatos a aldehídos aromáticos $\alpha, \beta$-insaturados, empleando la catálisis vía ión iminio como modo de activación del enal. El catalizador utilizado es el $\mathrm{O}$ trimetilsilildiarilprolinol 17, conocido coloquialmente como catalizador de Jørgensen (Esquema 10), y los resultados de la reacción dependieron del disolvente utilizado. Aunque la reacción no es diasteroselectiva cuando se emplean malonatos asimétricamente sustituídos, los aductos de Michael obtenidos se utilizaron como precursores en la síntesis de (+) y (-) paroxetina y de (+) femoxetina (Jørgensen et al, 2006).

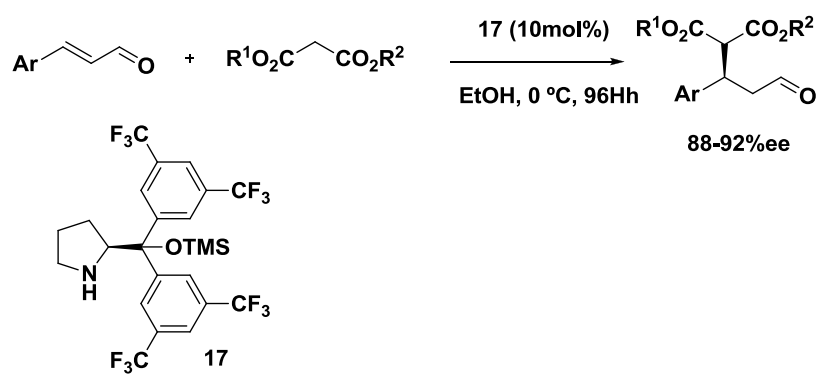

Figura 10. Adición conjugada de malonatos a enales.

El mismo grupo de investigación describió la primera adición conjugada enantioselectiva de $\beta$-cetoésteres a enales catalizadas por 17 tanto en medio acuoso como sin disolvente, obteniendo, en un proceso "one-pot", ciclohex-2enonas 2,5-disustituídas con excelentes excesos enantioméricos (Figura 11). El proceso transcurre a través de la formación de un aducto de Michael intermedio que experimenta una secuencia de decarboxilación/ciclación/deshidratación promovida por el ácido $p$-toluensulfónico ( $p$ - $\mathrm{TsOH})$ que actúa como cocatalizador, favoreciendo tanto el proceso de hidrólisis del 
éster como la reacción aldólica intramolecular y su final eliminación (Carlone et al, 2006).

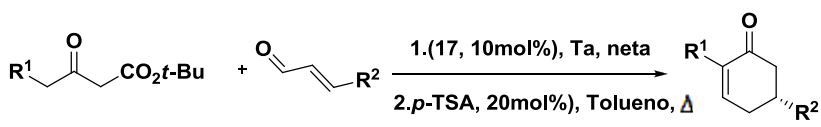

Figura 11. Síntesis enantioselectiva de ciclohex-2-enonas 2,5disustituídas.

Más recientemente, en 2008, Barbas III describió la utilización de trifluoroetil tioésteres como nucleófilos en las adiciones de Michael organocatalíticas (Esquema 12), estableciendo que los tioésteres, con un pKa menor de 16.9, son nucleófilos adecuados para ser activado por bases y ser utilizados en organocatálisis. Propone además que si se utiliza una cetona con un pKa $>17$ en sus protones a la reacción requiere una activación por formación de una enamina (Alonso et al, 2008).

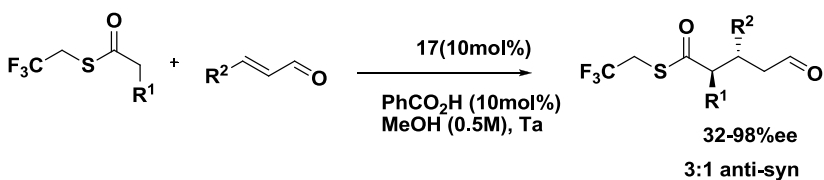

Figura 12. Tioésteres como nucleófilos en adiciones de Michael.

De esta manera, y teniendo en cuenta el límite propuesto por Barbas III, se han descrito algunos procesos donde se usan $p$-nitrobencilmetilcetonas como nucleófilos. Los autores sugieren que la presencia del grupo $p$-nitrofenilo convierte a la acetona en un excelente nucleófilo en procesos organocatalíticos, siendo capaz de reaccionar con enales. Inicialmente se generan mezclas equimolares de 3hidroxiciclohexanonas epímeras en el carbono que soporta el $\mathrm{OH}$ en un proceso Michael/Aldol. Estas hidroxiciclohexanonas se transforman, en el medio de la reacción, en $\alpha$-arilciclohexenonas por adición de $\mathrm{p}-\mathrm{TsOH}$, con buenos rendimientos, ee de hasta el $96 \%$ y una completa diastereoselectividad (Figura 13) (Duce et al, 2011).

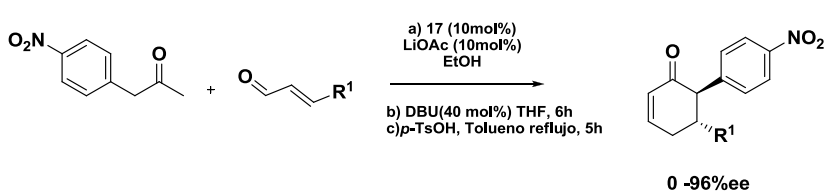

Figura 13. Síntesis enantioselectiva de $\boldsymbol{\alpha}$-arilciclohexenonas.

Del mismo modo, arilacetonitrilos pueden participar como nucleófilos versátiles en adiciones de Michael a aldehídos $\alpha, \beta$-insaturados, mediante la incorporación de un grupo nitro en el anillo de fenilo en posiciones orto y para, que actúa como grupo activante incrementando la acidez en la posición bencílica (Figura 14). La secuencia adición de Michael/reducción con $\mathrm{NaBH}_{4} /$ lactonización permite la obtención de lactonas disustituídas diastereoméricamente puras y con elevados rendimientos químicos y pureza óptica (Cid et al, 2010). Aprovechando la acidez intrínseca del carbono bencílico, la mezcla de diasteroisómeros obtenida en la adición de Michael se epimeriza al diastereoisómero más estable en el sustrato cíclico (Figura 14).<smiles>[R]C=CC=O</smiles><smiles>[R]C(CC=O)C(C#N)c1ccccc1</smiles>

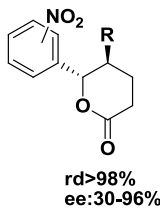

Figura 14. Nitrofenilacetonitrilos como nucleófilos en adiciones de Michael.

Este mismo grupo estudia la distinta evolución que pueden experimentar los aductos de Michael obtenidos a partir de $\beta$-cetosulfonas con aldehídos alifáticos $\alpha, \beta$ insaturados catalizadas por $\mathbf{1 7}$ al variar las condiciones de reacción (Alemán et al, 2010) (Figura 15). Establecen que la versatilidad de los aductos de Michael hace posible su uso en procesos tándem y one-pot, obteniendo ciclos polisustituídos con múltiples estéreocentros. La acidez de los protones en a al grupo sulfona hace imposible la obtención de un solo diastereoisómero en el producto de adición de Michael (Figura 15). Asimismo, los intentos de obtener derivados de 3-hidroxiciclohexanonas en un proceso "onepot" se limitan con que los productos dependen de la naturaleza de la cetona de partida. En general, se forman mezclas equimolares de diastereoisómeros en el C-3, pero si $\mathrm{R}^{2}$ es un grupo bencilo la reacción continúa formándose derivados de ciclohexenonas con excelentes enantioselectividades en un proceso tándem Michael/aldol/deshidratación en condiciones ácidas.

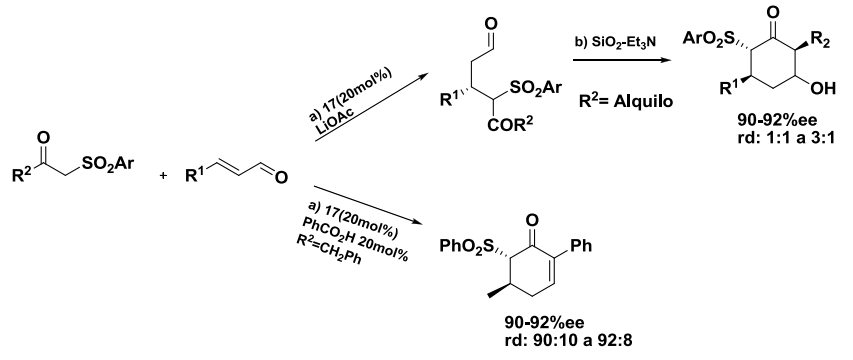

Figura 15. Versatilidad de los aductos de Michael.

Un ejemplo de la versatilidad de los aductos de Michael 1,5-dicarbonílicos, es el publicado por Jorgensen $y$ colaboradores y se resume en el Figura 16 (Marigo et al, 2006). La utilización de un 4-cloro cetoéster como nucleófilo proporciona clorhidrinas en un proceso dominó Michael/Aldol catalizado por $\mathbf{1 7}$ y acetato sódico como aditivo. El tratamiento posterior, en un proceso "one-pot", con una base promueve una $\mathrm{S}_{\mathrm{N}} 2$ intramolecular conduciendo a epoxicetonas polisustituídas con excelentes ee. Éstas se transforman en distintos derivados de alfa? ?eta-epoxi ciclohexanonas en posteriores transformaciones. 


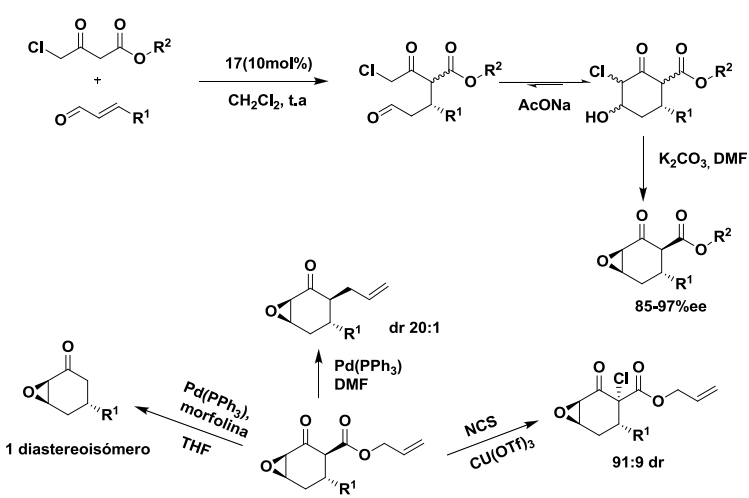

Figura 16. Reacción One-Pot Michael/Darzens.

Hayashi describe también una síntesis de 2ciclohexenonas 2,5,6-trisustituídas enantiopuras por reacción de 3-oxo-glutaratos catalizada por 18 (Oterbutildimetilsilildifenilprolinol). Aunque el proceso es descrito por los autores como una cicloadición formal [3+3], se trata de un proceso tándem Michael/Knoevenagel y posee la limitación de que únicamente se emplean enales con sustituyentes aromáticos en la posición beta. (Hayashi et al, 2009) Figura 17.

$$
\begin{aligned}
& \mathrm{RO}_{2} \mathrm{C} \rightleftharpoons \mathrm{CO}_{2} \mathrm{R}+\mathrm{O}^{1} \stackrel{\begin{array}{l}
18(10 \mathrm{~mol} \%) \\
\mathrm{PhCO}_{2} \mathrm{H}(20 \mathrm{~mol} \%)
\end{array}}{\mathrm{CH}_{2} \mathrm{Cl}_{2} \mathrm{ta}} \\
& \overbrace{\mathrm{H}}^{\mathrm{Ph}} \overbrace{18}^{\mathrm{Ph}}
\end{aligned}
$$

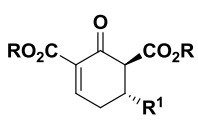$$
\text { 94-99\%ee }
$$

Figura 17. Cicloadición formal [3+3].

En 2011, Pericás y colaboradores describieron la síntesis de derivados de ciclohexenona como los descritos por Hayashi, pero utilizando como catalizador $O$ trimetilsilildifenilprolinol 19 inmovilizado sobre poliestireno, en la reacción del 3-oxoglutarato con enales aromáticos y alifáticos. Además implementaron un proceso de flujo que permite la producción continua de estos compuestos enantioenriquecidos, reciclando el catalizador en al menos seis ciclos sin pérdida en la actividad ni en la enantioselectividad de los productos obtenidos (Figura 18). (Pericas et al, 2011)

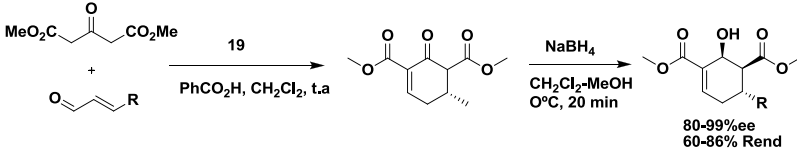

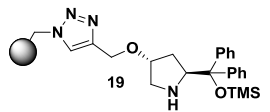

Figura 18. Síntesis de derivados de ciclohexano catalizadas por 19.

Hasta el momento se han descrito los diferentes tipos de metilenos activos que se han utilizado en las adiciones conjugadas organocatalíticas a aldehídos $\alpha, \beta$-insaturados, estableciendo que en la mayoría de los casos los aductos de
Michael se transforman en derivados de ciclohexenonas ya sea por procesos tándem o "one-pot”.

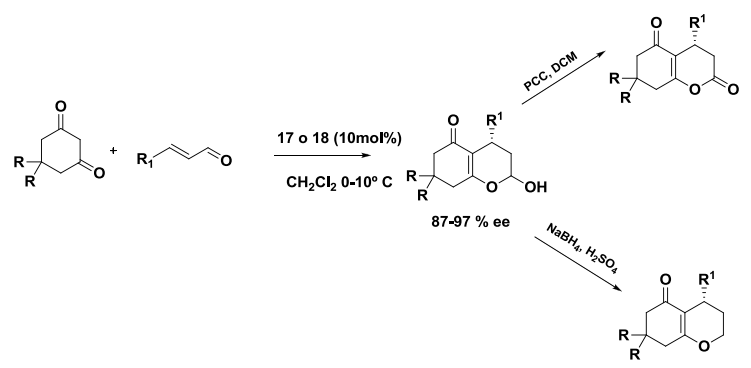

Figura 19. Reacción dominó Michael/Heterociclación.

Sin embargo Rueping y Jørgensen casi simultáneamente en 2008 describieron procesos donde, utilizando 1,3dicetonas cíclicas como nucleófilos en la adición de Michael, se obtienen hemiacetales cíclicos en un proceso dominó Michael/heterociclación que pueden ser transformados en cromanonas (Rueping et al, 2008) para el caso de Rueping (Figura 19) y en 3,4-dihidropiranos (Franke et al, 2008) con excelentes ee, pero pobre diastereoselectividad para el trabajo publicado por Jørgensen (Figura 20).

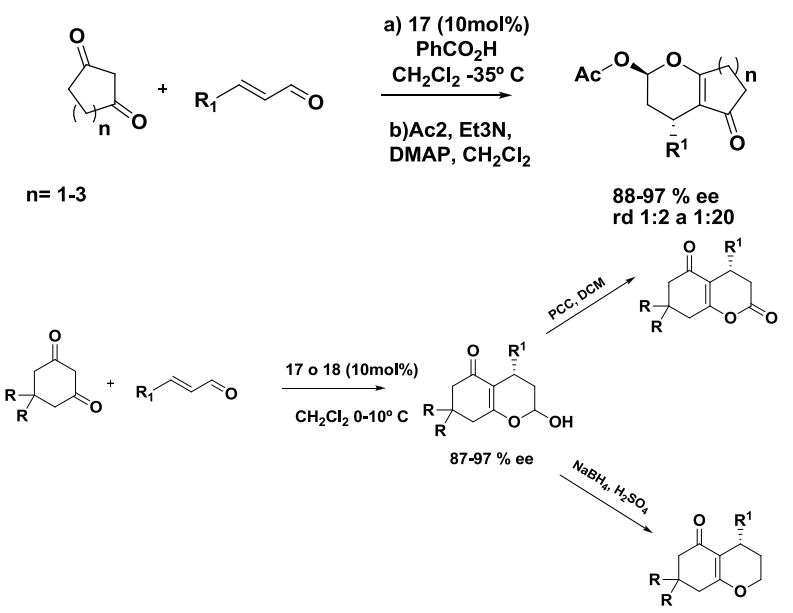

Figura 20. Síntesis asimétrica de 3,4-dihidropiranos.

Un ejemplo similar fue descrito en 2014 por (GuevaraPulido et al, 2014) donde en un proceso Tandem-one-pot, se obtienen 3,4-dihidro piranonas con excelentes excesos enantiómericos utilizando enales tanto alifáticos como aromáticos (Figura 21).

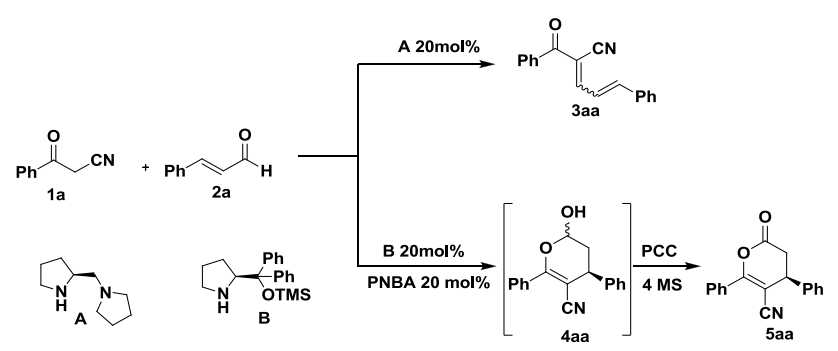

Figura 21. 3,4-dihidro piranonas por una metodología Tándem-OnePot. 
Esté mismo grupo investigación ha descrito una secuencia organocatalítica One-Pot, donde fue posible enlazar tres procesos como son: Adición de Michael - Oxido reducción de Tishchenko y Lactonización con total diastereoselectividad y excelente enantioselectividad (Guevara-Pulido et al, 2014) Figura 22.

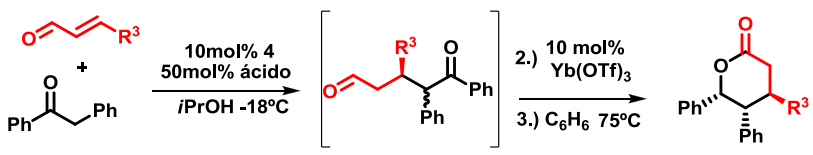

Figura 22. "One-Pot” Michael-Tishchenko-Lactonización (Catalizador B Figura 21).

Recientemente fue publicada la primera síntesis enantioselectiva de carbociclos y heterociclos de siete miembros por organocatálisis obteniendo un control total de la enantioselectividad (Guevara-Pulido et al, 2016). Figura 23.

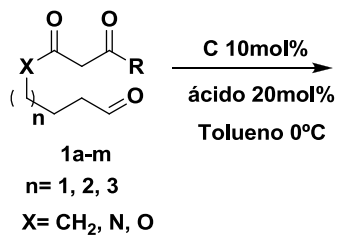

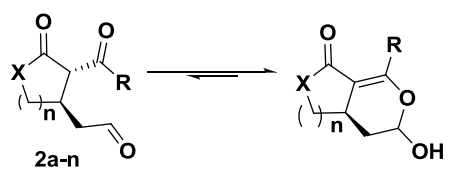

$\mathbf{R}=\mathrm{OBn}, \mathrm{Ph}, \mathrm{Me}, \mathrm{p}-\mathrm{MePh}, \mathrm{p}-\mathrm{OMePh}$

Figura 23. Síntesis enantioselectiva de carbociclos y heterociclos por catálisis vía ión iminio.

\section{Ciclo catalítico común catálisis vía ión iminio}

Todas las reacciones descritas a lo largo de esta apartado han sido catalizadas por los derivados de difenil y diarilprolinol y ocurren sobre aldehídos $\alpha, \beta$-insaturados. Independientemente del producto final de la reacción todas pasan por un producto intermedio común que es un aducto de Michael.

De esta manera se puede describir un ciclo catalítico común (Nielsen et al, 2011) para todas las adiciones conjugadas, que consiste en la formación de un ión iminio 3 entre 17 o 18 y el aldehído a, $\beta$-insaturado 4, que favorece el ataque al carbono- $\beta$ por parte del nucleófilo 5 por la cara Re menos impedida del ión iminio, formando la enamina 6 . Ésta isomeriza en el medio de reacción al ión iminio 7 , que se hidroliza obteniendo el aducto de Michael 8 y regenerando el catalizador de partida (Figura 24).

\section{Catálisis somo}

La catálisis SOMO (orbital molecular ocupado por un solo electrón), se basa en la idea de que la oxidación de un electrón de una enamina rica en electrones genera selectivamente un catión radical reactivo con tres electrones $\pi$ (Macmilan, 2008). La electrofilicidad de este orbital, permite que reaccione fácilmente con una variedad de bases débilmente nucleófilas en el carbono a de la enamina obteniéndose productos de alquilación. La aplicación de este

principio en un sistema catalítico que usa aminas secundarias quirales y un oxidante adecuado de un electrón, ha tenido un gran éxito, ya que permite la apertura de nuevas vías para catalizar la a-funcionalización asimétrica de compuestos que contienen carbonilo (Figura 25).

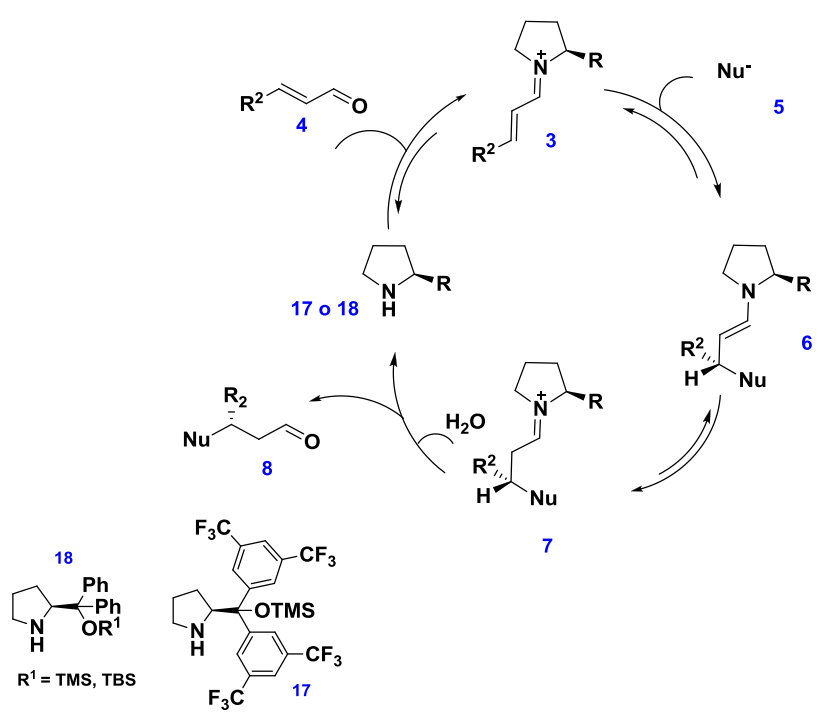

Figura 24. Ciclo catalítico de la catálisis vía ión iminio. (Nielsen et al, 2011).

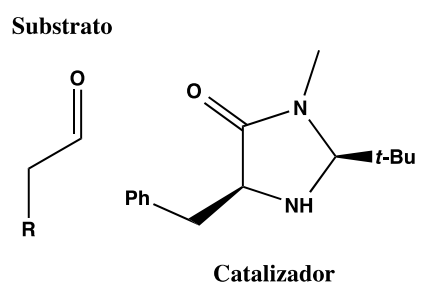

Catalizador

alfa-alilación

alfa-enolación

Figura 25. Modo de reacción vía catálisis SOMO (Macmilan, 2008).

\section{Catálisis por contra-ión}

Jacobsen desarrollo una nueva forma de activación organocatalítica, que dirige adiciones altamente enantioselectivas en forma transitoria generada en iones $\mathrm{N}$ acil-iminio y oxocarbenio (Raheem et al, 2008). En este sistema, los catalizadores quirales de tiourea, que son conocidos por formar complejos fuertes electrostáticamente al unirse con los iones de haluro, ionizan los enlaces carbonocloro débiles de las cloroamidas y cloroacetales para generar un par de iones transitorios. El complejo catalizador de cloruro aniónico resultante, funciona como un contra-ión quiral, obteniendo el ataque de nucleófilos por una sola cara del enantiómero. Los resultados iníciales son notables debido a que las fuerzas que actúan a través del espacio, en lugar de a través de enlaces, son suficientes para transferir la 
estereoquímica del catalizador al sustrato con alta fidelidad (Figura 26).

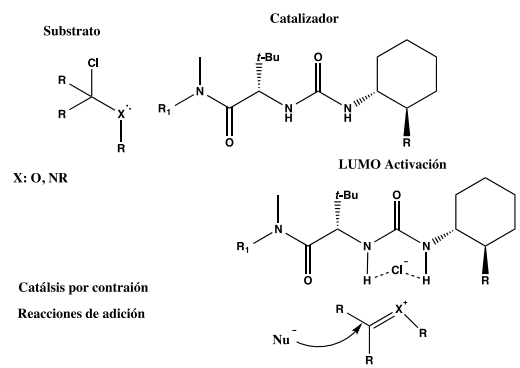

Figura 26. Modo de reacción vía contraión (Macmilan, 2008).

\section{Ejemplos destacados}

Recientemente se ha descrito un proceso donde, utilizando el derivado de prolinol C, fue posible catalizar enantioselectivamente la adición conjugada de Michael de aldehídos a enonas cíclicas vía enamina. En este estudio fue posible utilizar una gran variedad tanto de aldehídos como de enonas cíclicas obteniendo buenas enantio- y diastereoselectividades, lo que contribuye a sintetizar prostaglandinas de manera simple. (Figura 27)

$$
\text { (10lueno }
$$

Figura 27. Adición vía enamina de aldehídos a enonas cíclicas (Scarpino-Schietroma, 2011).

Otro ejemplo a destacar es la versatilidad del catalizador bifuncional 16, un derivado de diaminometilenmalononitrilo, en la adición conjugada de malonatos a cetonas $\alpha, \beta$ insaturadas en ausencia de disolvente. Este catalizador, cuya estructura recuerda a la de una urea bifuncional, proporciona los correspondientes aductos de Michael con buenos rendimientos químicos y excelentes enantioselectividades (Figura 28) (Hirashima et al, 2014).

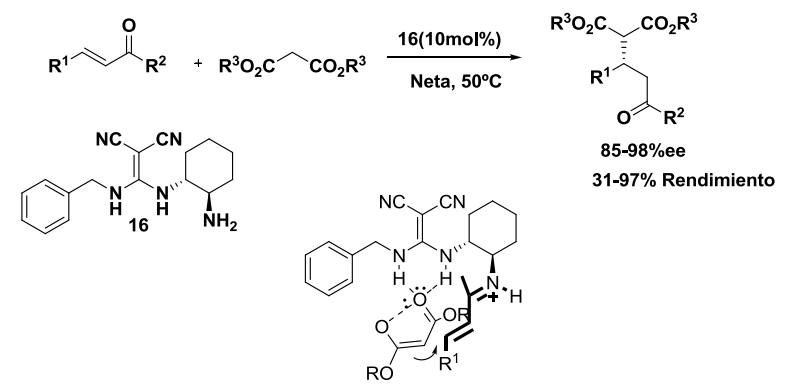

Figura 28. Adición conjugada de malonatos a enonas utilizando un catalizador bifuncional (Hirashima et al, 2014).
La catálisis procede a través de un estado de transición análogo al propuesto por Kwiatkowski y colaboradores. (Dudzinski et al, 2012) El grupo amino primario del catalizador condensa con la enona formando un ión iminio intermedio que activa la posición $\beta$. Simultáneamente, los dos protones ácidos del catalizador interaccionan con el oxígeno carbonílico del malonato estabilizando el enolato, y favoreciendo la aproximación del enolato del malonato por la cara Re del ión iminio (Figura 28).

Por último no se puede dejar de lado uno de los métodos más usado por la industria farmacéutica para la síntesis a grna escala de fármacos que es la resolución cinética dinámica (DKR), que siendo una evolución de la KR (resolución cinética), ocurre siempre y cuando el racemato de partida pueda epimerizar o racemizar fácilmente en las condiciones de reacción (Figura 29). Esta resolución se ha llevado a cabo por catálisis organometálica (Prévost et al, 2010), enzimática (Yasukawa et al, 2011) y recientemente por organocatálisis (Pellissier 2011).

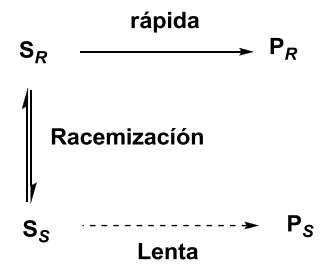

Figura 29. Resolución Cinética Dinámica (DKR) (Faber, 2001).

Si bien es un método más eficiente para la preparación de compuestos enantioméricamente puros, su efectividad depende de la facilidad de epimerización de los compuestos a desracemizar.

Cabe resaltar que en América latina se han presentado muy pocas investigaciones con respecto al campo sintético asimétrico catalizado por moléculas orgánicas. No obstante, a inicios de la década de los 90 y hasta estos últimos años, se han publicado varios trabajos acerca de nuevos métodos de síntesis enantioselectiva de $\beta$-aminoácidos y otros compuestos usando aminas quirales como catalizadores, realizadas por Juaristi y su grupo de investigación en México (Juaristi y Hernandez, 2011) (Figura 30).

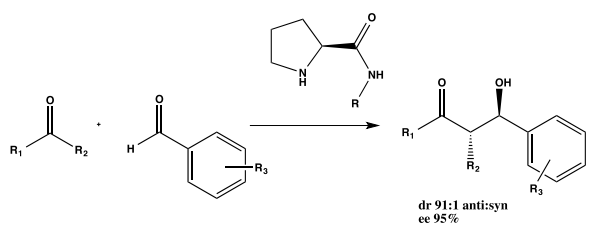

Figura 30. (S)-prolina-(S)-fenilalanina (Juaristi y Hernandez, 2011) Reaccion aldólica organocatalizada.

\section{CONCLUSIONES}

No se puede desconocer que cada una de las tres metodologias descritas en este artículo, ha contribuido al entendimiento de la síntesis asimétrica y antes de escoger una de ellas como la más prominente se debe considerar que 
son complementarias. Sin embargo, La organocatálisis se basa en el empleo de pequeñas moléculas orgánicas (sin metales), normalmente quirales, para catalizar transformaciones orgánicas. Presenta importantes ventajas en comparación con los otros tipos de catálisis asimétrica, tales como la simplicidad de las condiciones de reacción, no siendo necesarios medios inertes ni el empleo de disolventes secos. Algunos de los organocatalizadores son disponibles comercialmente a bajo costo, otros son fácilmente accesibles a partir de sustancias naturales o pueden ser sintetizados de manera sencilla en el laboratorio. Además, al no tener metales en su estructura evitan posibles problemas de contaminación, por lo que son tolerables en la producción de fármacos, alimentos y derivados agroquímicos. en America Latina el conocimiento de este tipo de metodologías es escaso, tan solo un reducido número de grupos de investigación enfocan sus estudios a resolver este tipo de problemas de interes mundial. Esperamos que esta revisión ofrezca una alternativa de investigación a grupos Colombianos y Latinoamericanos que esten interesados en aportar a la síntesis asimetrica de principios activos de medicamentos.

\section{AGRADECIMIENTOS}

Los autores agradecen a la Vicerrectoria de Investigación de la Universidad El Bosque, Bogotá-Colombia la financiación del proyecto $\mathrm{PCl}$-2015-8218.

\section{REFERENCIAS}

- Alemán J. Marcos V. Marzo L J. L. García Ruano L J. 2010. Influence of the Reaction Conditions on the Evolution of the Michael Addition of $\beta$-Keto Sulfones to $\alpha, \beta$-Unsaturated Aldehydes. European Journal Organic Chemistry, 23: 4482-4491.

- Alza E. Sayalero S. Cambeiro X, C. Martín-Rapún R, P. Miranda R. Pericàs M A. 2011. Catalytic Batch and Continuous Flow Production of Highly Enantioenriched Cyclohexane Derivatives with Polymer-Supported Diarylprolinol Silyl Ethers. SynLett, 4: 464-468

- Alonso D A. Kitagaki S. Utsumi N. Barbas III C F. 2008. Towards organocatalytic polyketide synthases with diverse electrophile scope: trifluoroethyl thioesters as nucleophiles in organocatalytic Michael reactions and beyond. Angewandte Chemie International Edition, 47: 4588-4591

- Beyer H. y Walter W. 1987. Manuel de Química Orgánica. Alemania: Reverte. 200.

- Brandau S. Landa A. Franzen J. Marigo M. Jørgensen K. A. 2006. Organocatalytic Conjugate Addition of Malonates to $\alpha, \beta$-Unsaturated Aldehydes: Asymmetric Formal Synthesis of (-)-Paroxetine, Chiral Lactams, and Lactones. Angewandte Chemie International Edition, 45: 4305-4309

- Carlone A. Marigo M. North C. Landa A. Jørgensen K. A. 2006. A simple asymmetric organocatalytic approach to optically active cyclohexenones. Chemical Communications, 47: 4928-4930

- Cid M, B. Duce S. Morales S. Rodrigo E. García-Ruano J. L. 2010. Nitrophenylacetonitriles as Versatile Nucleophiles in Enantioselective Organocatalytic Conjugate Additions. Organic Letters, 12: 3586-3589

- Corey E. y Grogan M. 1999. Enantioselective synthesis of amino nitriles from $\mathrm{N}$-benzhydryl imines and HCN with chiral bicycly guanidine as catalyst. Organic Letters, 1: 157-160.

- Dang T. y Kagan H. 1971. The asymmetric synthesis of hydratropic acid and amino-acids by homogeneus catalytic hydrogenation. Journal of the Chemical Society D: Chemical Communications. 481.

- Denmark S. Crudden C. Matsuhashi H. 1997. Catalytic epoxidation of alkenes with oxone-2-fluoro ketones. The Journal of Organic Chemistry, 62: 82888289.
- Duce S. Jorge M. Alonso I. Garcia-Ruano J. L. Cid M. B. 2011. An organocatalytic approach to enantiomerically enriched aarylcyclohexenones and cyclohexanones. Organic Biomolecular Chemistry, 9: $8253-8260$

- Dudzinski K. Pakulska A M. Kwiatkowski P. 2012. An efficient organocatalytic method for highly enantioselective michael addition of malonates to enones catalyzed by readily accessible primary amine-thiourea. Organic Letters, 14: 4222-4225

- Eder U. Suaer G. Wiechert R. 1971. Patente nº DE 2014757. German.

- Faber. K. 2001. Non-sequential processes for the transformation of a racemate into a single stereoisomeric product: proposal for stereochemical classification. Chemistry European Journal, 7: 5004-5010

- Franke P. T. Richter B. Jørgensen K. A. 2008. Organocatalytic Asymmetric Synthesis of Functionalized 3,4-Dihydropyran Derivatives. Chemistry European Journal, 14: 6317-6321

- Guevara-Pulido J. Andrés J. M. Pedrosa R. 2014. Organocatalytic Domino Michael-Heterocyclization Reaction of $\alpha, \beta$-Unsaturated Aldehydes and $\alpha$ Cyano Ketones: Synthesis of Enantioenriched 4,5,6-Trisubstituted 3,4 Dihydropyranones. European Journal Of Organic Chemistry, 36: 8072-8076

- Guevara-Pulido J. Andrés J. M. Pedrosa R. 2014. One-Pot Sequential Organocatalytic Michael-Tishchenko-Lactonization Reactions. Synthesis of Enantioenriched 4,5,6-Trisubstituted $\delta$-Lactones. Journal of Organic Chemistry, 79: 8638-8644

- Guevara-Pulido J. Andrés J. M. Avíla D. Pedrosa R. 2016. Enantioselective synthesis of seven-membered carbo- and heterocyles by organocatalyzed intramolecular Michael addition. RSC Advances, 6: 30166-30169

- Hajos Z. y Parrish, D. 1971. Patente n ${ }^{\circ}$ DE 2102623. German.

- Hayashi Y. Toyoshima M. Gotoh H. Ishikawa H. 2009. Diphenylprolinol Silyl Ether Catalysis in an Asymmetric Formal Carbo [3 + 3] Cycloaddition Reaction via a Domino Michael/Knoevenagel Condensation. Organic Letters, 11: $45-48$

- Hirashima S. Sakai T. Nakashima K. Watanabe N. Koseki Y. Mukai K. Kanada Y. Tada N. Itoh A. Miura T. 2014. Solvent-free asymmetric conjugate addition of malonates to enones using a diaminomethylenemalononitrile organocatalyst. Tetrahedron Letters. 55: 4334-4337

- Janice Y. G. Klunder M. Hanson R. H. Masamune H. Ko. S. Sharpless K. 1987. Catalytic asymmetric epoxidation and kinetic resolution. Journal of American Chemical Society, 109: 5765-5780

- Juaristi E. Hernandez J. 2011. Asymmetric Aldol Reaction Organocatalyzed by (S)-Proline-Containing Dipeptides: Improved Stereoinduction under Solvent-Free Conditions. Journal of Organic Chemistry 76:1464-1467

- Katsuki T. Sharpless K. B. 1980. The first practical method for asymmetric epoxidation . Journal of American Chemical Society, 102: 5974-5976

- Kitamura M. Ohkuma T. Inoue S. Sayo N. Kumobayashi H. Akutagawa S. 1988. Homogeneus asymmetric hydrigenation. Journal of American Chemical Society 629.

- Knowles W. S, Sabacky M. 1968. Catalytic asymmetric hydrogenation employing. Journal of the Chemical Society D: Chemical Communications, 1447-1449

- Knowles W. Sabacky M. Vineyard B. 1972. Catalytic asymmetric hydrogentation. Journal of the Chemical Society D: Chemical Communications, 10-11

- Kolb H. C. Van Nieuwenhze M. S. Sharpless K B. 1994. Catalytic Asymmetric Dihydroxylation. Chemical Review, 94: 2483-2547

- List B. Lerner R. Barbas C. 2000. Prolina-catalyzed direct asymmetric aldol reactions. Journal of American Chemical Society, 122: 2395-2396.

- Marigo M. Bertelsen S. Landa A. Jørgensen K. A. 2006. One-Pot Organocatalytic Domino Michael-Aldol and Intramolecular $\mathrm{S}_{\mathrm{N} 2}$ Reactions. Asymmetric Synthesis of Highly Functionalized Epoxycyclohexanone Derivatives. Journal of American Chemical Society,128: 5475-5479

- Macmilan D. 2008. The advent and development of organocatalysis. Nature, 455: 304-308

- Matsunaga S. Shibasa M. 2008. Multimetallic Bifunctional Asymmetric Catalysis. Bulletin Chemistry Society Japon, 81: 60-75

- Miyashita A. N. 1980. Synthesis BINAP. Journal of American Chemical Society, 102: 7932-7934

- Miller S. Copeland G. Papaioannou N. Horstmann T. Ruel E. 1998. Kinetic resolution of alcohols catalyzed by terpeptides containing the $\mathrm{N}$ alkylimidazole substructure. Journal of American Chemical Society, 120: 1629-1620 
- Nielsen M. Worgull D. Zweifel T. Gschwend B. Bertelsena S Jørgensen K. A. 2011. Mechanisms in aminocatalysis. Chemical Communications, 47: 632-649

- Noyori R. Hashiguchi S. 1997. Asymmetric transfer hydrogenation catalyzed by chiral ruthenium complexes. Accounts Chemical Research, 30: 97-102

- Noyori R, Kitamura M. 1991. Addition of Organometallic Reagents to Carbonyl Compounds. Angewandte Chemie International Edition, 30: 49-69

- Ohta T. Takata H. Kitamura M. Nagay K. N. 1987. Asymmetric hydrogenation of unsaturated carboxylic acids. Journal of Organic Chemistry, 52: 3174-3176

- Ohkuma T. Ooka H. Ikariya T. Noyori R. 1995. Preferential hydrogenation of aldehydes and ketones. Journal of Chemical Society, 117: 10417-10418

- Patel R. N. 2008. Synthesis of chiral pharmaceutical intermediates by biocatalysis. Coordination Chemical Review, 252: 659-701.

- Pellissier H. 2011. Organocatalyzed Dynamic Kinetic Resolution. Advanced Synthesys and Catalysis, 353: 659-676

- Perotti J. 2012. "Diseño, Síntesis Y Evaluación De Novedosos Ligandos Quirales Derivados De Taddol". Santa Fe, Argentina.

- Pizzano A. Carmona E. 2001. PREMIO NOBEL DE QUMICA 2001: Síntesis asimétrica catalítica. Anales de la Real Sociedad de Química, 50-55.

- Prévost S. Gauthir S. De Andrade M. C. C. Mordant C. Touati A. R. Lesot P. Savignac P. Ayad T. Phansavath P. Ratovelomanana-Vidal V. Genet J. P. 2010. Dynamic kinetic resolution of $\alpha$-chloro $\beta$-keto esters and phosphonates: hemisynthesis of Taxotere ${ }^{\circledR}$ through Ru-DIFLUORPHOS asymmetric hydrogenation. Tetrahedron: Asymmetry, 21: 1436-1446

- Raheem I. Thiara P. Peterson E. Jacobsen E. 2007. Enantioselective PictetSpenglertype cyclizations of hydroxylactams: $\mathrm{H}$-bond donor catalysis by anion binding. Journal Of American Chemical Society, 129 13404-13405

- Reisman S. Doyle A. 2008. Enantioselective thiourea-catalyzed additions to oxocarbenium ions. Journal Of American Chemical Society, 130: 7198-7199

- Reetz M. T. 2013. Biocatalysis in Organic Chemistry and Biotechnology: Past, Present, and Future. Journal Of American Chemical Society, 135: 12480-12496

- Reetz M. T. Wu S. Zheng H. Prasad S. 2010. Directed evolution of enantioselective enzymes: An unceasing catalyst source for organic chemistry. Pure Applied Chemistry, 82: 1575-1584
- Rueping M. Sugiono E. Merino E. 2008. Asymmetric Organocatalysis: An Efficient Enantioselective Access to Benzopyranes and Chromenes. Chemistry European Journal 14, 6329-6332

- Scarpino-Schietroma D M. Monaco M R. Visca V. Insogna S. Overgaard J. Bella M. 2011. Enamine-Mediated Addition of Aldehydes to Cyclic Enones. Advanced Synthesys and Catalisys, 2011, 353: 2648-2652.

- Shibasaki M. Kanai M. Matsunaga S. Kumagai. 2009. Recent progress in asymmetric bifunctional catalysis using multimetallic systems. Accounts Chemical Research. 42, 1117-1127

- Sigman M. Jacobsen E. 2000. Schiff base catalysts for the asimmetric Strecker reaction identified and optimized from parallel synthetic libraries. Journal Of American Chemical Society, 120: 4901-4902

- Takaya H. Ohta T. Sayo N. Kumobayashi H. Akutagawa S. Inoue S. 1987. Enantioselective hydrogenation of allylic and homoallylic alcohols. Journal Of American Chemical Society, 109: 1596-1597

- Tu Y. Wang Z. Shi Y. 1996. An efficient asymmetric epoxidation for transolefins mediated by a fructose derived ketones. Journal of American Chemical Society, 118: 9806-9807

- Vineyard B. D. Knowles W. Sabacky M. Bachman G. Weinkauff D. 1977 Asymmetric hydrogenation. Rhodium chiral bisphosphine catalyst. Journal Of American Chemical Society, 99: 5946-5952

- Yamada Y. M. Shibasaki M. 1997. Direct catalytic asymmetric aldol reactions of aldehydes with unmodified ketones. Angewandte Chemie International Edition, 36: 1871-1873

- Yang D. 1996. A C2 symmetric chiral ketones for catalytic asymmetric epoxidation of unfunctionalized oleofins. Journal of American Chemical Society, 118: 491-492

- Yasukawa K. Hasemi R. Asano Y. 2011. Dynamic Kinetic Resolution of aAminonitriles to Form Chiral a-Amino Acids. Advanced Synthesys and Catalisys. 353: 2328-2332. 